\title{
Gain Improvement of Dual Band Antenna Based on Complementary Rectangular Split-Ring Resonator
}

\author{
Noelia Ortiz, Francisco Falcone, and Mario Sorolla \\ Millimeter Wave Laboratory, Electrical and Electronic Engineering Department, Public University of Navarra, Arrosadía Campus, \\ 31006 Pamplona, Spain
}

Correspondence should be addressed to Mario Sorolla, mario@unavarra.es

Received 7 October 2011; Accepted 9 November 2011

Academic Editors: C. Luxey and J. K. Muppala

Copyright ( 2012 Noelia Ortiz et al. This is an open access article distributed under the Creative Commons Attribution License, which permits unrestricted use, distribution, and reproduction in any medium, provided the original work is properly cited.

A simple and successful dual band patch linear polarized rectangular antenna design is presented. The dual band antenna is designed etching a complementary rectangular split-ring resonator in the patch of a conventional rectangular patch antenna. Furthermore, a parametric study shows the influence of the location of the CSRR particle on the radiation characteristics of the dual band antenna. Going further, a miniaturization of the conventional rectangular patch antenna and an enhancement of the complementary split-ring resonator resonance gain versus the location of the CSRR on the patch are achieved. The dual band antenna design has been made feasible due to the quasistatic resonance property of the complementary split-ring resonators. The simulated results are compared with measured data and good agreement is reported.

\section{Introduction}

The possibility of obtaining media with simultaneously negative permeability and permittivity was hypothesized by Veselago in the late 1960s [1]. In spite of the interesting properties presented by such media, it was not until 2000 that the first experimental evidence of a medium with simultaneously negative permeability and permittivity was demonstrated [2]. The original medium proposed in [2] consists of a bulky combination of metal wires and split-ring resonators (SRRs) [3].

The SRR electromagnetic properties have been already analyzed in $[4,5]$. This analysis shows that the SRR behaves as an LC resonant tank that can be excited by an external time-varying magnetic field applied parallel to the particle axis, thus producing a quasi-static resonant effect [4]. Therefore, the SRR has subwavelength dimensions at its quasistatic resonance, allowing very compact device designs. Up to now, these self-resonant particles have been used in the design of microwave filters in planar technology $[6,7]$. However, in this paper, we have taken advantage of the complementary split-ring resonator (CSRR) concept [8] to design a miniaturized dual band patch antenna with vertical polarization, also studying how to improve radiation efficiency for the resonance produced by the CSRR in this kind of antennas. The CSRR is inspired on Babinet principle [9], and, as occurs with the SRR, it also exhibits a quasi-static resonance, which enables the particle to be electrically small $[8,9]$.

Up to now, the use of metamaterial concepts in practical miniaturized antennas is a very challenging research topic [10-16] and the achieved results based on self-resonance particles as SRRs or CSRRs [16] exhibit low radiation efficiencies driving to low-gain antennas comparing to the results of the parametric study presented in this paper. In this sense, the excitation of a CSRR etched in the patch of a conventional patch antenna allows us to design dual band patch antennas. Going further, the presence of the CSRR etched in some positions of the path also produces a miniaturization of both patch antenna resonances, leading to miniaturized dual band antennas. Replacing the CSRR within a slot of its same external dimensions, the slot does not exhibit a resonance at the same frequency of the CSRR, but at higher frequencies and more than one slot should be placed in the patch depending on their position in order to achieve a dual band response. The resonance frequency of a rectangular slot on a dielectric is approximately given by $c_{\text {light }} /(a+b) \cdot \sqrt{\varepsilon_{r}}$, where $c_{\text {light }}, \varepsilon_{r}, a$, and $b$ are the speed of light in vacuum, dielectric relative permittivity, and the external dimensions 
of the rectangular iris, respectively. In opposition to the resonance frequency of a rectangular slot, the resonant frequency of the CSRR is much lower for the same physical size. Hence, it can be designed to exhibit a resonance at lower frequencies comparing to different shapes of slots that can also be etched in a conventional patch. The design presented in this paper gives an alternative solution to the existing dual band antenna designs [17], as the ones carried out by loading a rectangular patch antenna with a pair of bent slots or embedded step slots close to the patch nonradiating edges, or the ones done by spur lines or shorted microstrip antenna with rectangular patch. Overall, as it can be shown in this paper, the properties of the CSRR allow us to design dual band miniaturized antennas based on the anisotropic properties of the CSRR as indicated by the measurements of a fabricated prototype and the parametric studies of the presented design in opposition to dual band antennas produced by the radiation of slots, whose resonances are dependent on their physical length. The prototype has been designed to exhibit a dual band behaviour in two frequency bands in the range from $4 \mathrm{GHz}$ to $5 \mathrm{GHz}$ for wireless applications. Nowadays, there is a growing trend to integrate different wireless communication systems in one single user terminal as long as to reduce the overall size. Since all these systems work at different frequency bands, dual and multiband antennas with frequency ratios around 1.2 between different bands are desirable. For this application, the type of antennas presented in this paper are a good alternative as introducing different CSRRs on the patch multiband antennas can be obtained very easily taking as a starting point the dual band antennas presented in this paper. The dual band antenna design presented has been validated, and a parametric study of the CSRR location and its influence on antenna radiating characteristics is presented and analyzed.

\section{CSRR Excitation in a Rectangular Patch}

The excitation of CSRRs has been usually driven by an incident electric field normal to the particle plane. In order to understand the excitation of these particles, let us consider the CSRR presented in Figures 1(b) and 1(c). Comparing the excitation of an SRR with a CSRR, the CSRR particle should be rotated $90^{\circ}$ from the position of the SRR particle, as it is shown in Figure 1(a). Following the theoretical discussion shown in $[4,5]$, the operation of the SRR near its first resonance frequency obeys the effect of resonant polarizabilities, which gives the resonant magnetic and electric dipolar moments $m_{z}, p_{x}$, and $p_{y}$ as a function of the exciting field components $B_{z}^{\text {inc }}, E_{x}^{\text {inc }}$, and $E_{y}^{\text {inc }}$. Complementarily, using the Babinet principle [9], the CSRR can be excited by the incident complementary fields $E^{\text {incc }}$ and $B^{\text {incc }}$, which are related to $E^{\text {inc }}$ and $B^{\text {inc }}$ by $E^{\text {incc }}=c \cdot B^{\text {inc }}$ and $B^{\text {incc }}=-(1 / c)$. $E^{\text {inc }}$ by means of another set of resonant polarizabilities, thus given an electric dipole $p_{z}^{c}$ and magnetic dipoles $m_{x}^{c}$ and $m_{y}^{c}$. Then, as seen in Figures $1(\mathrm{~b})$ and 1(c), depending on the CSRR position inside the patch, it will be excited by incident electric field normal to the particle plane $\left(E_{z}\right)$ and by incident magnetic field tangent to the particle plane $\left(B_{x}\right)$. For the excitation of the CSRR by the magnetic field $\left(B_{x}\right)$, the
CSRR should be rotated $90^{\circ}$ in the patch as it is shown in Figure 1(c) comparing to the orientation of the CSRR in Figure 1(b).

In order to show graphically the excitation of the CSRR by the existing fields inside a rectangular patch antenna, Figures 2 and 3 show the electric fields and surface current distributions at the resonance frequency of the CSRR for the locations of this particle according to the layouts of Figures 2(a) and 3(a). In Figure 2, the CSRR particle has been located for its proper excitation by $E_{z}$ electric field component, placed in one position inside the area where $E$ field distributions are higher. Otherwise, in Figure 3, the CSRR particle has been located where magnetic field distributions are more concentrated, for the best suitable CSRR excitation by $B_{x}$ magnetic field component. Though, for this case, the CSRR is also excited by $E_{z}$. The simulations of the structures presented in this paper have been performed with the commercial finite-integration time-domain CST Microwave Studio Code.

\section{Parametric Study}

The parametric study carried out in this work shows how the position of the CSRR (without changing its dimensions) has influence on the radiation efficiency of both antenna resonances for the orientation of the particle as it is in Figure 1(b). On this way, for some locations of the CSRR in the patch, radiation efficiencies up to $50 \%$ are achieved for both resonances. These results together with the measurement results of the prototype comparing to the simulated results show the usefulness of this kind of dual band patch antennas. The simulated antenna in the parametric study has the same dimensions as the fabricated one, excluding the placement of the CSRR inside the patch, which varies from Pos $=-9 \mathrm{~mm}$ to Pos $=9 \mathrm{~mm}$ in the $y$-axis direction and from $u=1 \mathrm{~mm}$ to $u=13 \mathrm{~mm}$ in the $x$-axis direction. In Figure 1(b), the references of both Pos and $u$ parameters are specified. The substrate employed in the simulated and fabricated prototype is the commercially available Arlon 250LX-0193-43-11 ( $\varepsilon_{r}=2.43$ and thickness $\left.h=0.49 \mathrm{~mm}\right)$. The physical width and length of the rectangular patch antenna are $18.43 \mathrm{~mm}$ and $23.68 \mathrm{~mm}$, respectively [18]. Then, its resonance has been set around $5 \mathrm{GHz}$. The width of the micro strip line is $1.34 \mathrm{~mm}$, corresponding to a characteristic impedance of $50 \Omega$. This line exhibits an offset from the centre of the patch antenna in order to match its reflection coefficient at its working frequency. The offset is $(X 1=$ $-13.37 \mathrm{~mm}$ and $Y 1=0 \mathrm{~mm}$ ) (see Figure $1(\mathrm{~b})$ ).

The CSRR particle has been designed to exhibit its quasistatic resonance frequency below the resonance frequency of the patch, obtaining more compact devices highlighting the advantages of the resonance properties of anisotropic particles comparing to other slots already used for dual band antenna designs. The radiation produced by conventional slots does not have the same origin comparing to the radiation produced by a CSRR particle, and their electric length should be longer comparing to the electric length of the CSRR. The necessary physical dimensions of the CSRR to achieve a radiation frequency below the resonant frequency 

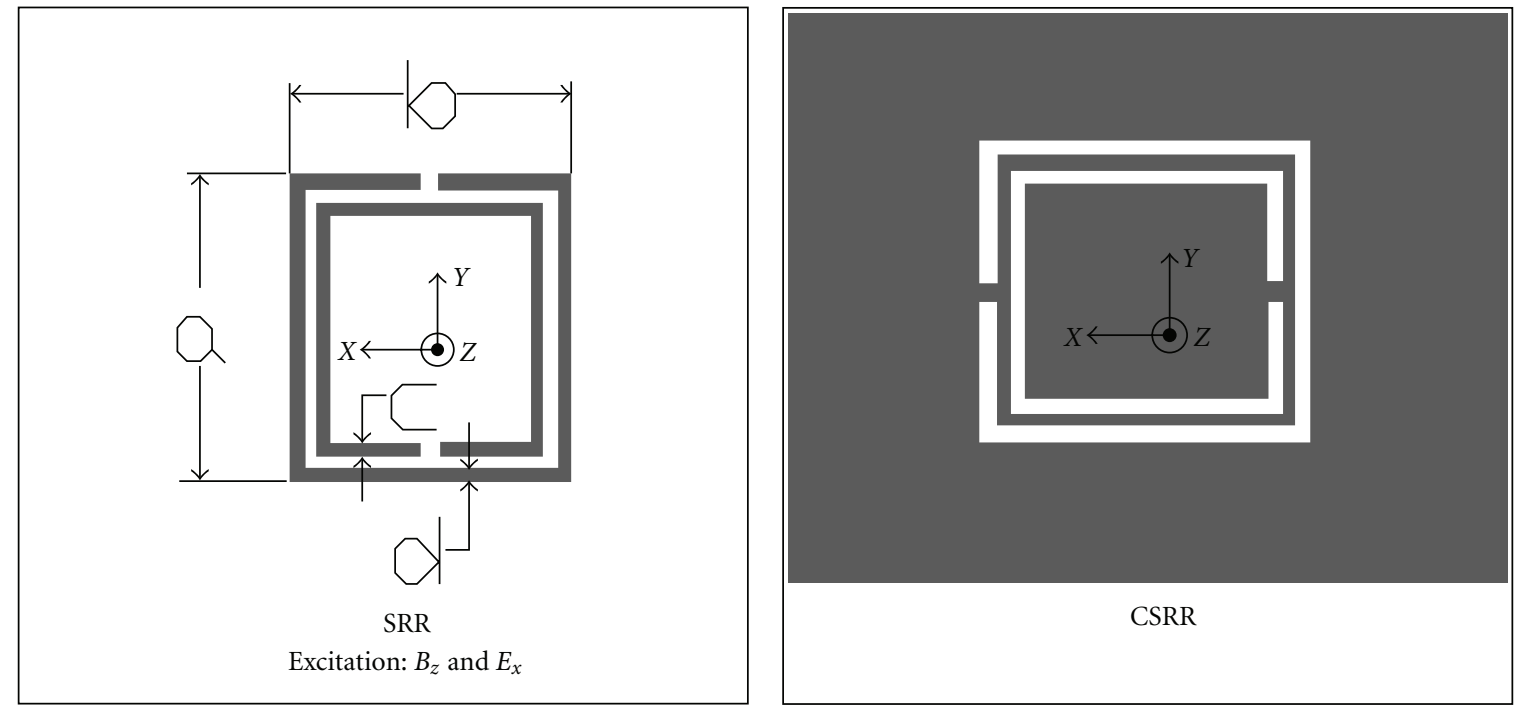

(a)

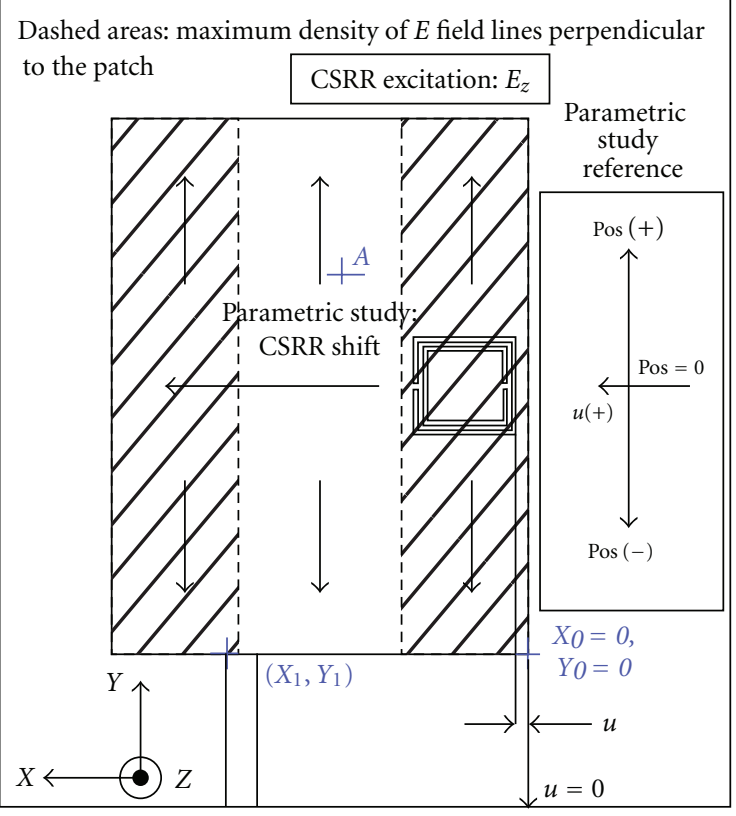

(b)
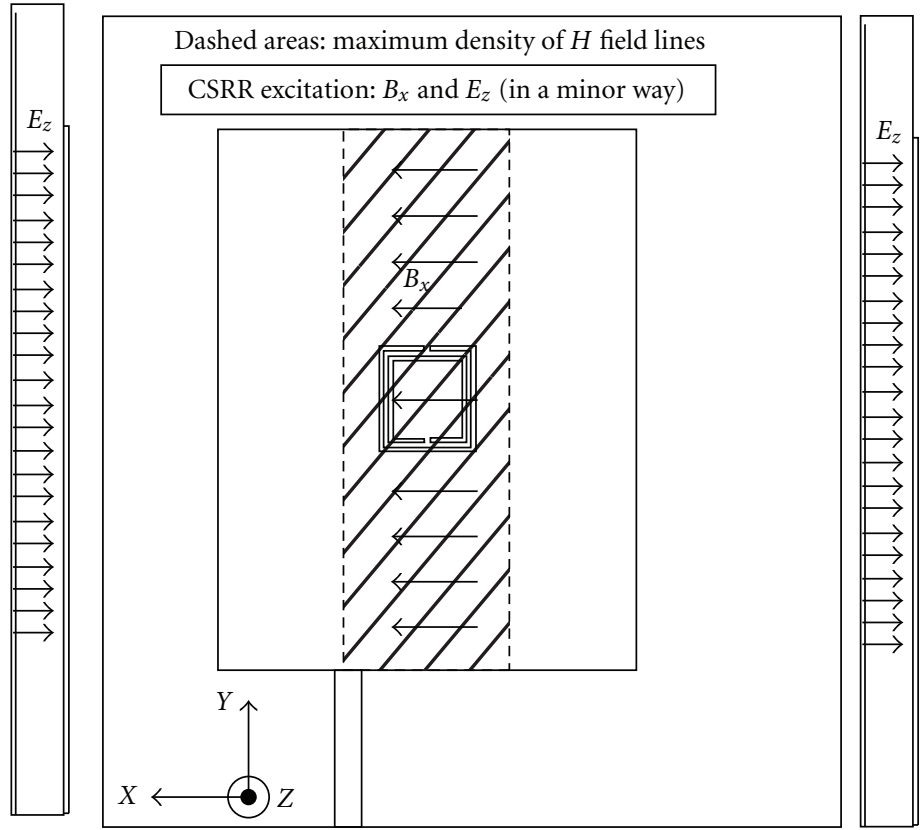

(c)

FIGURE 1: (a) SRR and CSRR topologies relevant dimensions. (b) Configuration of dual band CSRR-rectangular patch antenna. CSRR excitation by incident electric field normal to the particle plane, $E_{z}$. (c) Configuration of dual band CSRR-rectangular patch antenna. CSRR excitation by magnetic field tangent to the particle plane, $B_{x}$, and by electric field normal to the particle plane, $E_{z}$.

of the designed rectangular patch have been calculated using the design formulas for SRR reported in [4], resulting in this case in $a=4.6 \mathrm{~mm}, b=4.2 \mathrm{~mm}$, and $c=d=0.2 \mathrm{~mm}$. In all the results presented in this paper, the first resonance of the dual band antenna is the one produced by the excitation of the CSRR, while the second resonance is produced by the conventional patch itself.

In Figures 4(a) and 4(b) radiation efficiencies as results from the parametric study are shown for both resonances. Figure 4(a) shows how the radiation efficiency of the resonance produced by the excitation of the CSRR increases while the anisotropic particle is placed at the radiating edges of the rectangular patch (Pos parameter values of $-9 \mathrm{~mm}$ and $9 \mathrm{~mm}$ ). However, there are some positions for the CSRR inside the patch where the radiation due to the CSRR is cancelled. These positions correspond to values around Pos parameter of $0 \mathrm{~mm}$ and values around this value. This means that when the CSRR is etched in the centre of the rectangular patch its resonance is cancelled leading to a single resonance antenna.

Figure 4(b) shows how the radiation efficiency of the resonance produced by the patch decreases for some 


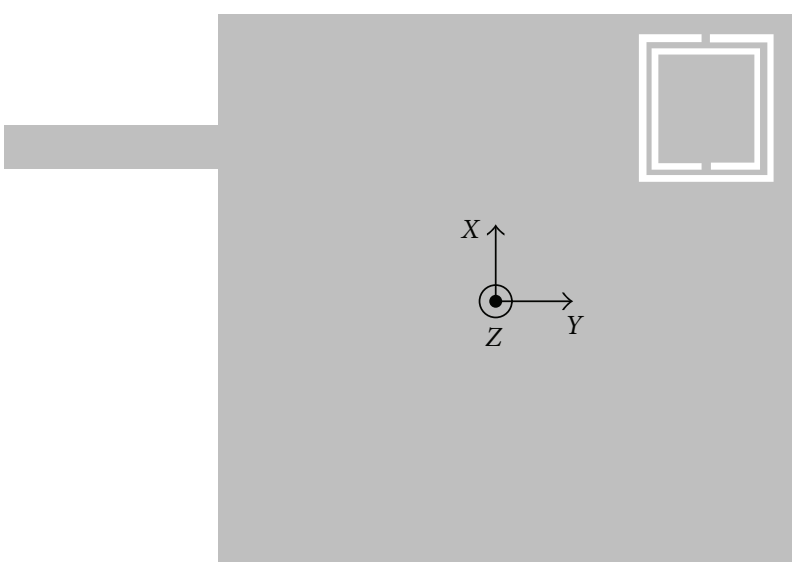

(a)

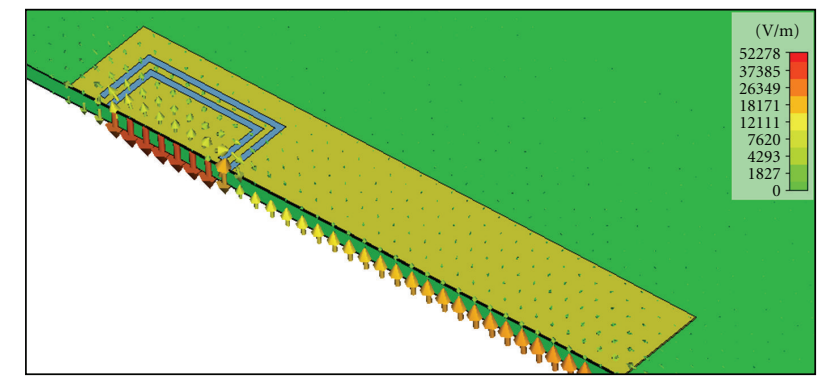

(b)

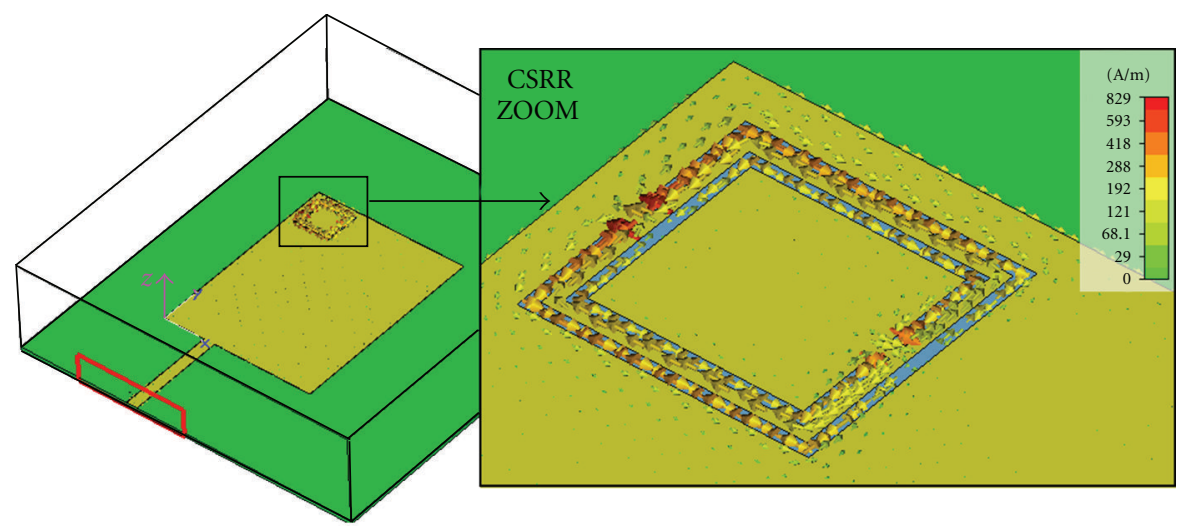

(c)

Figure 2: (a) Top view of the layout for $E$ field and current distributions analysis in reference with Figure 1(b). (b) Simulated $E_{z}$ field distribution in the CSRR at its resonant frequency. (c) Simulated current distributions in the CSRR at its resonant frequency.

$u$ positions when the CSRR is placed at the radiating edges of the conventional patch antenna. However, there are other $u$ positions at these edges where both resonances exhibit radiation efficiencies up to $50 \%$. The position values where radiation efficiency has been set to zero mean that the resonance produced by the CSRR particle or by the patch has been cancelled as explained before. The results of the parametric study for radiation efficiencies of both resonances show that the results are not symmetric, these differences are due to the asymmetric microstrip line excitation of the rectangular patch.

In Figures 5(a) and 5(b) gain values for $u$ parameter values $(u=3,4,11,12$, and 13$)$, which drive to the highest radiation efficiencies for both resonances, are depicted. The discontinuities with no values in the curves of Figures 5(a), 6(a), and 6(b) are because there is no resonance of the CSRR for those positions.

For the locations where the CSRR is etched around the centre of the rectangular patch, $u=6 \mathrm{~mm}, u=7 \mathrm{~mm}$, and $u=8 \mathrm{~mm}$, both resonances, the one produced by the CSRR and the one produced by the patch, are shifted to lower frequencies, resulting in a miniaturization of both frequency bands of dual band patch antenna comparing to a conventional rectangular patch antenna of the same dimensions.
By contrast, as the CSRR moves away from the centre of the patch towards the nonradiating edges (in $u$ direction for all its Pos parameter values), the resonance produced by the rectangular patch shifts to higher frequencies comparing to the resonant frequency of the conventional rectangular patch itself. In Figures 6(a) and 6(b) the resonant frequencies for the first and second resonances versus Pos parameter and $f 2 / f 1$ ratio are shown for $u=1, u=6$, and $u=13$ parameter values, where $f 1$ and $f 2$ are the resonance produced by the CSRR and the one produced by the rectangular patch, respectively. The miniaturization ratio of this type of antennas based on this design is around 1.2, but it also depends on the position of the CSRR on the patch, as the resonance frequencies are shifted. This behaviour is clearly shown in Figures 6(a) and 6(b).

From Figures 6(a) and 6(b) the miniaturization factor has been calculated for two different positions of the CSRR on the patch. In the first case, for particle location parameters of $u=13 \mathrm{~mm}$ and Pos $=-9 \mathrm{~mm}$ the miniaturization factor is 1.16 , corresponding to a radiation efficiency of $45.37 \%$ and gain of $3.59 \%$. For the second case, the position parameters values are $u=6 \mathrm{~mm}$ and Pos $=0 \mathrm{~mm}$. In this case the resonance produced by the CSRR has been cancelled and a single-band antenna is achieved. The miniaturization factor 


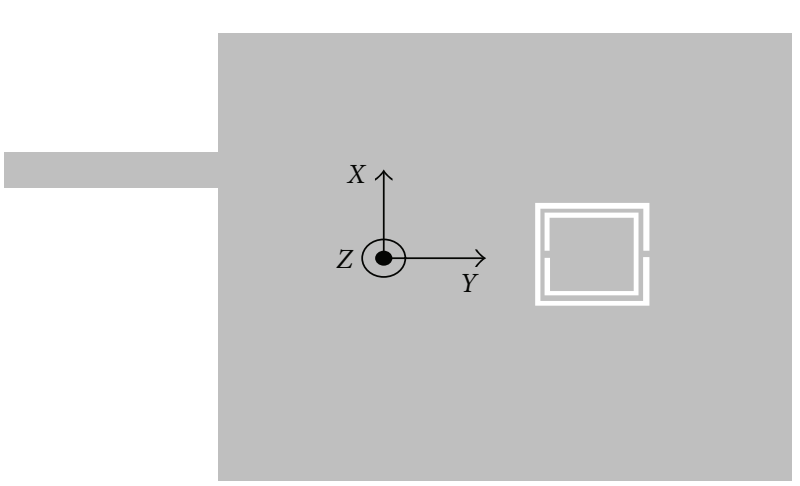

(a)

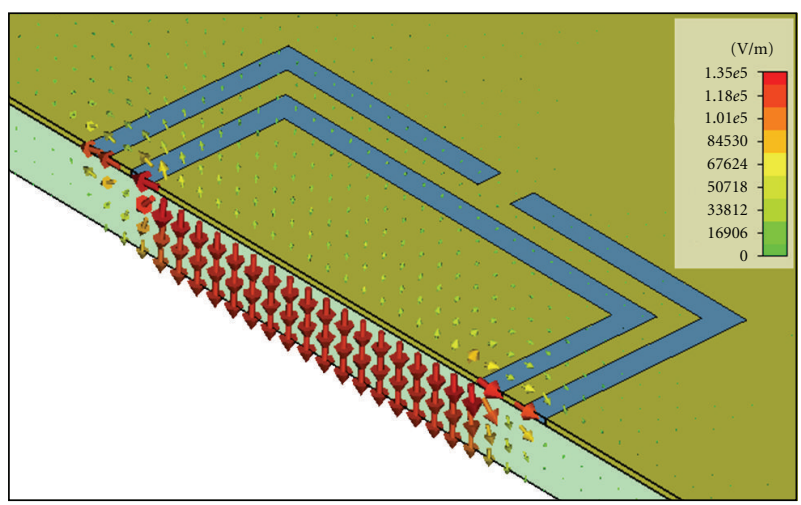

(b)

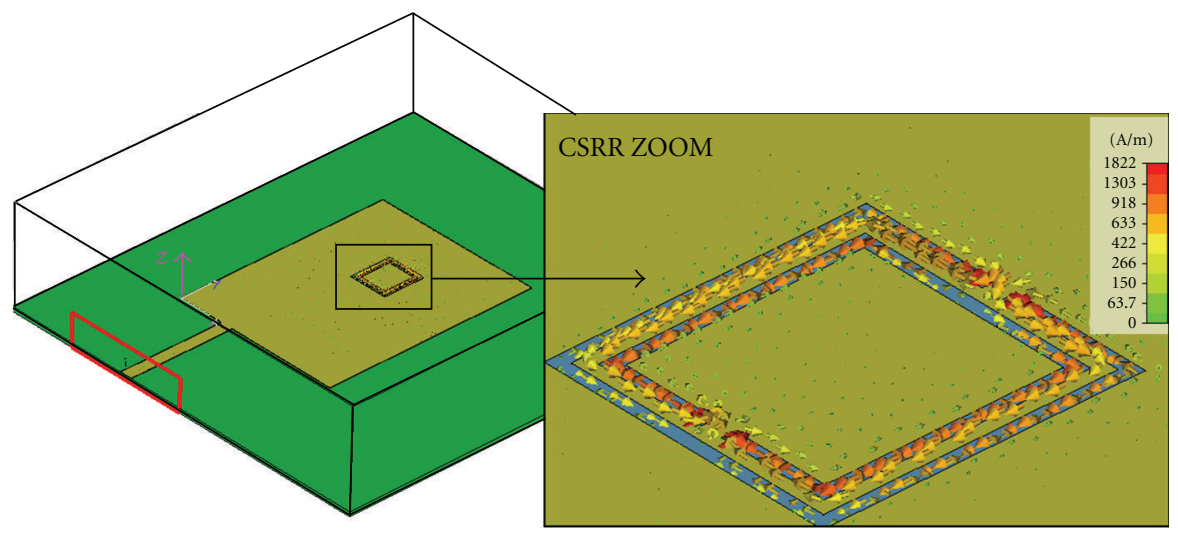

(c)

Figure 3: (a) Top view of the layout for $E$ field and current distributions analysis in reference with Figure 1(c). (b) Simulated $E_{z}$ field distribution in the CSRR at its resonant frequency. (c) Simulated current distributions in the CSRR at its resonant frequency.

is 1.1 , corresponding to a radiation efficiency of $84.36 \%$ and gain of $6.56 \mathrm{~dB}$.

\section{Experimental Results}

In order to demonstrate the usefulness of this dual band antenna design, a prototype has been fabricated. The fabricated prototype has been chosen from the parametric study in a case where the radiation efficiency is low comparing to the highest values obtained of this parameter. This case has been chosen to validate the usefulness of this design in a worst case condition. The prototype has been fabricated using a laser drilling machine. The design parameters of the CSRR particle are $a=4.6 \mathrm{~mm}, b=4.2 \mathrm{~mm}$, and $c=d=$ $0.2 \mathrm{~mm}$ (the same ones of those of the CSRR used in the parametric study). In the fabricated prototype the CSRR has been placed at A point (see Figure 1(b)) being the coordinates of this point $(X=-9.3 \mathrm{~mm}, \mathrm{Y}=15.54 \mathrm{~mm})$.

In Figure 7 simulated and measured reflection coefficient results of the fabricated prototype are shown. For matching measurements data has been collected by using an HP8510 network analyzer. As it can be seen in Figure 7, there is a frequency shift of $162 \mathrm{MHz}$ to lower frequencies for the lower resonance. The upper resonance presents a frequency shift of $84 \mathrm{MHz}$, shifted to lower frequencies. Although there is a frequency shift between simulated and measured results, the matching values achieved are properly predicted by simulations. The discrepancies between simulated and measured results are due to the manufacturing process as the CSRR manufacturing tolerances are critical, changing slightly its frequency resonance. In simulations, materials have been simulated considering their corresponding finite conductance and substrate has been simulated considering its dielectric losses. In Figure 8 a picture of the fabricated prototype is shown.

In Figures 9(a) and 9(b) measured results for normalized gain radiation patterns for $0^{\circ}$ and $90^{\circ}$ phi cut planes for both resonant frequencies are shown. Besides, simulated results just for $90^{\circ}$ phi cut are shown. No more simulated cuts are introduced to maintain the figures legible. These frequencies are $F 1=4.19 \mathrm{GHz}$ and $F 2=4.808 \mathrm{GHz}$, the resonant frequencies produced by the CSRR particle and conventional patch, respectively. The radiation pattern measured results show the feasibility of this type of dual band antenna design. Both resonances show cross-polar levels around $-20 \mathrm{~dB}$ for theta $0^{\circ}$.

Table 1 shows a comparison between simulated and measured results of the fabricated prototype from impedance matching and radiation point of views. This table shows that both resonances have similar characteristics. From matching 


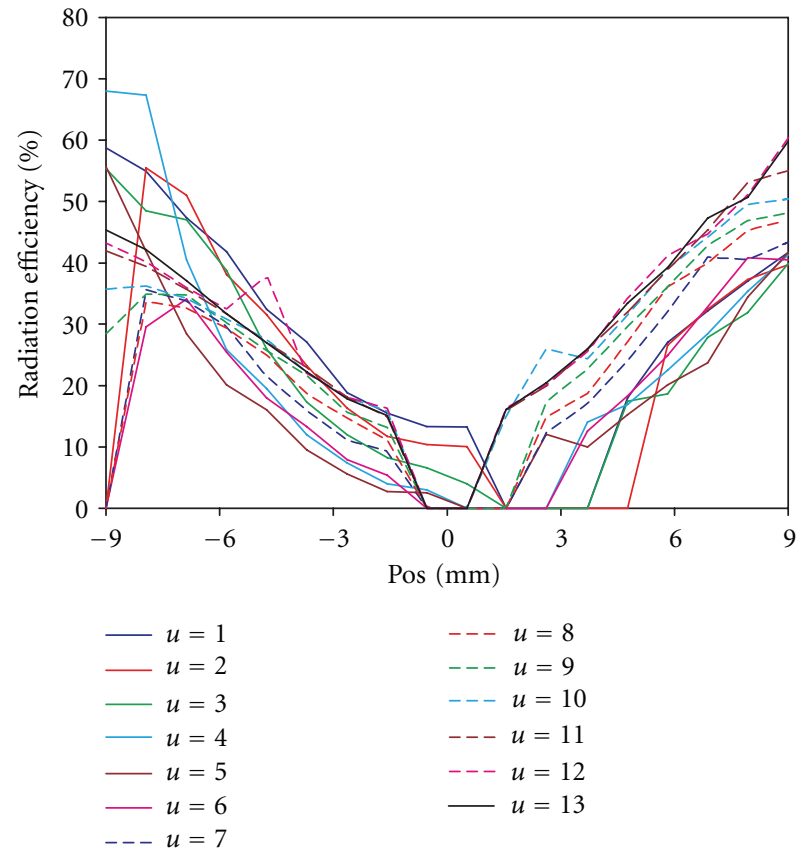

(a)

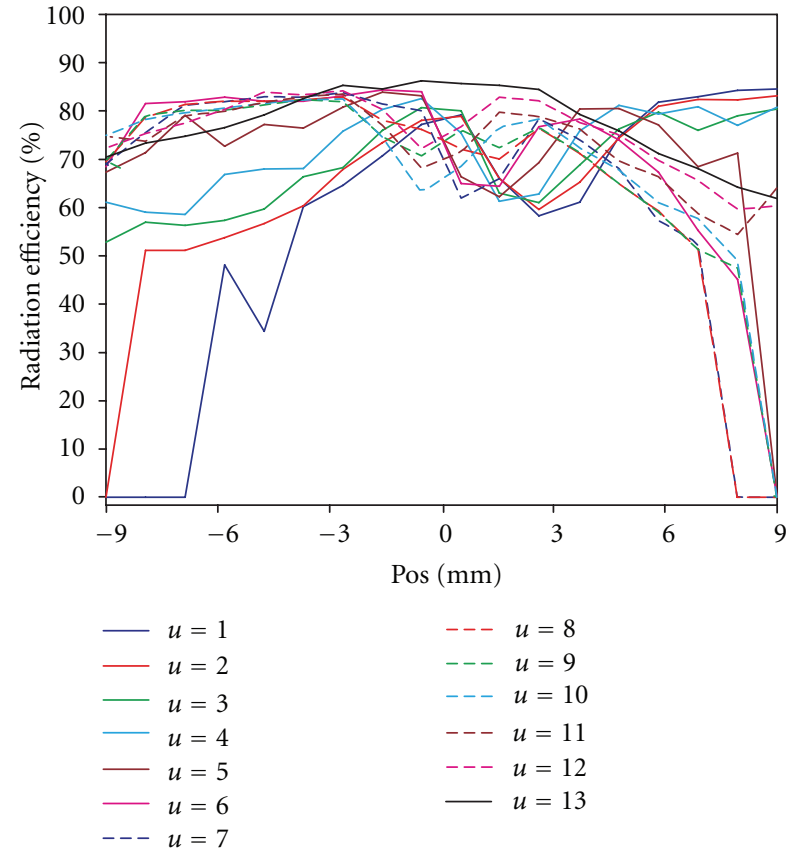

(b)

FIGURE 4: (a) Simulated radiation efficiency for the resonance produced by the CSRR. (b) Simulated radiation efficiency for the resonance produced by the rectangular patch antenna.

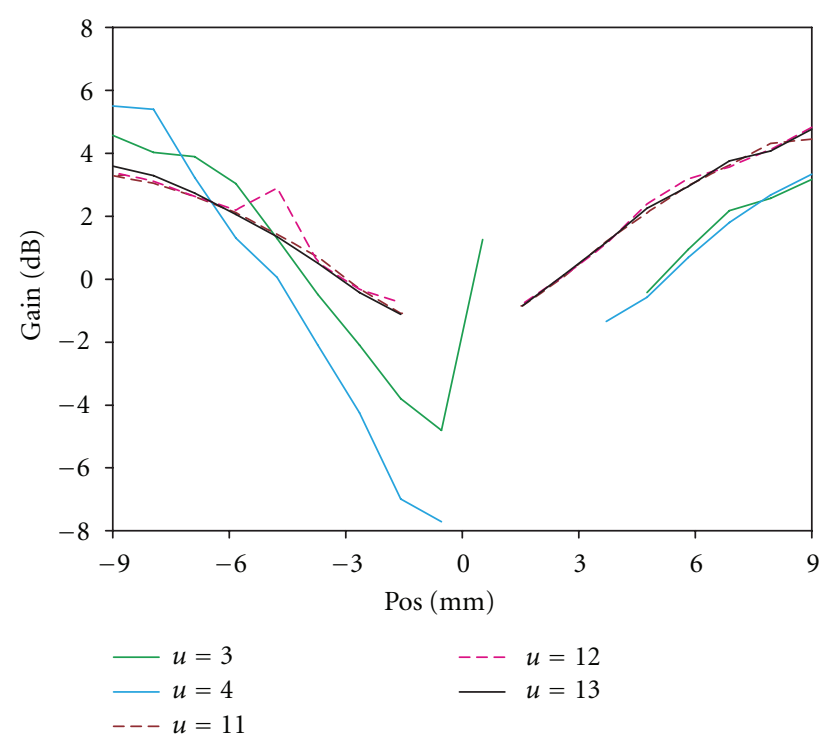

(a)

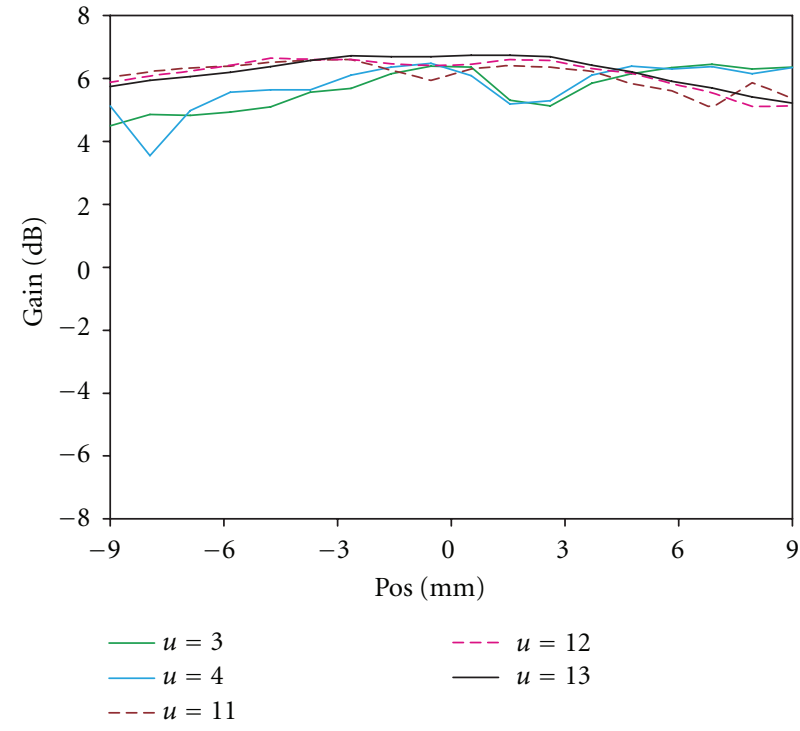

(b)

FIGURE 5: (a) Simulated gain values for the resonance produced by the CSRR. (b) Simulated gain values for the resonance produced by the rectangular patch antenna.

point of view, the parameters shown in Table 1 are reflection coefficient values for both resonant frequencies. From radiation point of view, parameters shown in Table 1 are peak directivity, peak gain, and radiation efficiency. Directivity values have been calculated [18] from measured gain radiation patterns for both resonances in order to calculate efficiency and verify the good agreement between simulated and measured data. Within the fabricated prototype the parametric study made in this work has been validated due to the good agreement between simulations and measured results. Although gain and radiation efficiency obtained by the resonance produced by the CSRR is low comparing to the second resonance in the fabricated prototype, these values are in accordance with the simulated results. Furthermore, 


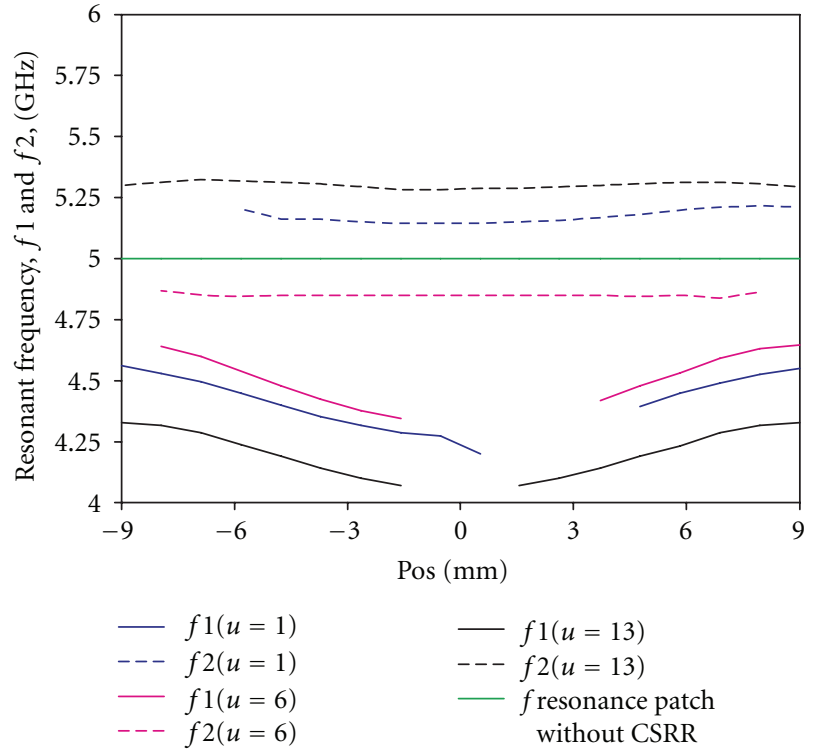

(a)

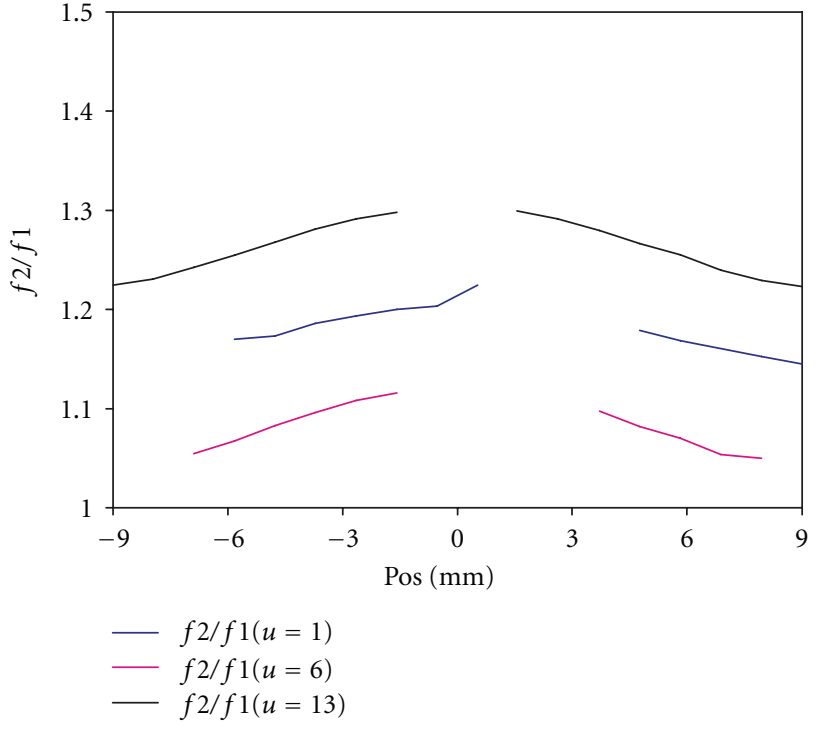

(b)

FIgure 6: (a) Parametric study results. $f 1$ and $f 2$ resonant frequencies. (b) Parametric study results. $f 2 / f 1$ ratio.

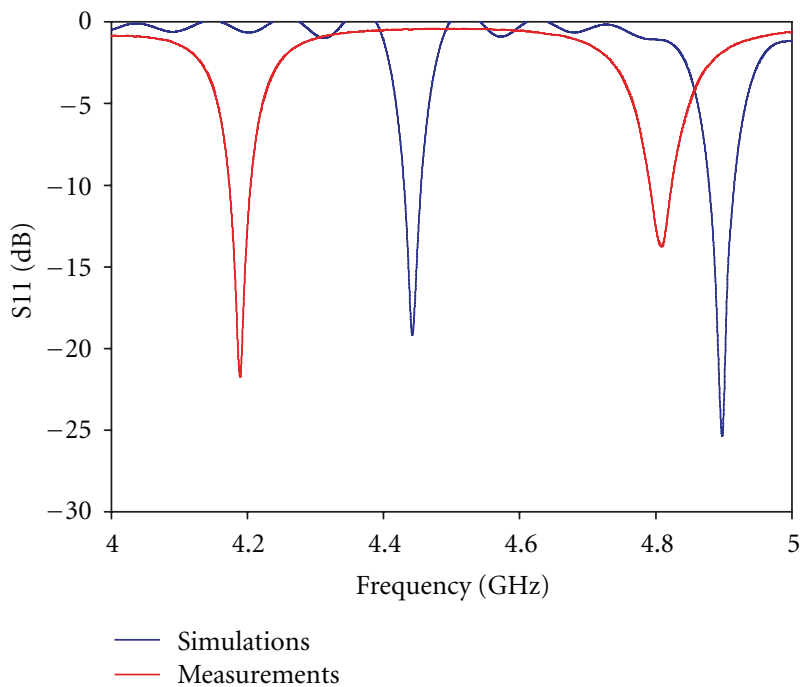

FIGURE 7: Simulated and measured reflection coefficient results.

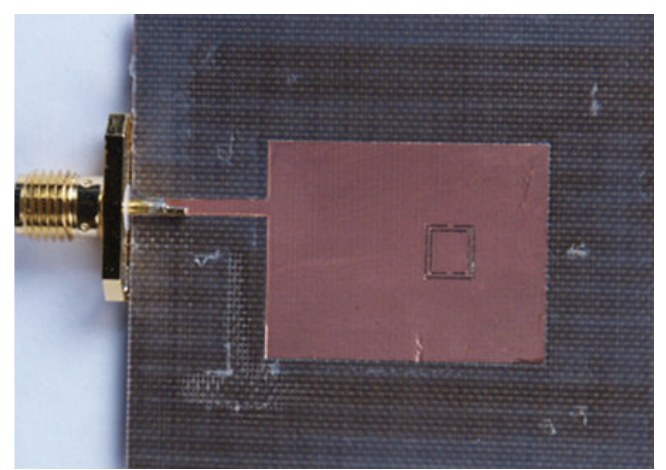

FIGURE 8: Fabricated prototype.
TABle 1

\begin{tabular}{|c|c|c|}
\hline Parameters & \multicolumn{2}{|c|}{ Matching characteristics } \\
\hline \multicolumn{3}{|c|}{ First resonance $(F 1)$} \\
\hline$F 1,(\mathrm{GHz})$ & 4.352 & 4.19 \\
\hline Reflection coefficient, (dB) & -16.75 & -21.25 \\
\hline $\begin{array}{l}\text { Effective bandwidth at } \\
-5 \mathrm{~dB}(\%)\end{array}$ & 1.60 & 1.52 \\
\hline \multicolumn{3}{|c|}{ Second resonance $(F 2)$} \\
\hline$F 2,(\mathrm{GHz})$ & 4.892 & 4.808 \\
\hline Reflection coefficient, $(\mathrm{dB})$ & -15.15 & -13.86 \\
\hline $\begin{array}{l}\text { Effective bandwidth at } \\
-5 \mathrm{~dB}(\%)\end{array}$ & 1.79 & 1.72 \\
\hline \multicolumn{3}{|c|}{ Radiation characteristics } \\
\hline \multicolumn{3}{|c|}{ First resonance $(F 1)$} \\
\hline Peak directivity, $(\mathrm{dBi})$ & 7.16 & 7.36 \\
\hline Peak gain, $(\mathrm{dB})$ & -0.97 & -0.11 \\
\hline Radiation efficiency (\%) & 15.38 & 17.92 \\
\hline \multicolumn{3}{|c|}{ Second resonance $(F 2)$} \\
\hline Peak directivity, $(\mathrm{dBi})$ & 7.243 & 7.74 \\
\hline Peak gain, $(\mathrm{dB})$ & 5.946 & 5.85 \\
\hline Radiation efficiency, (\%) & 74.18 & 64.83 \\
\hline
\end{tabular}

it is remarkable that gain values obtained for the positions of the CSRR in the patch studied in this work are higher compared to previous works [16].

Up to this point, in order to compare the performances of the dual band antenna topology presented in this paper with those of the same conventional patch antenna without a CSRR etched in its centre, in Table 2 measured data of the conventional rectangular patch antenna is shown. 


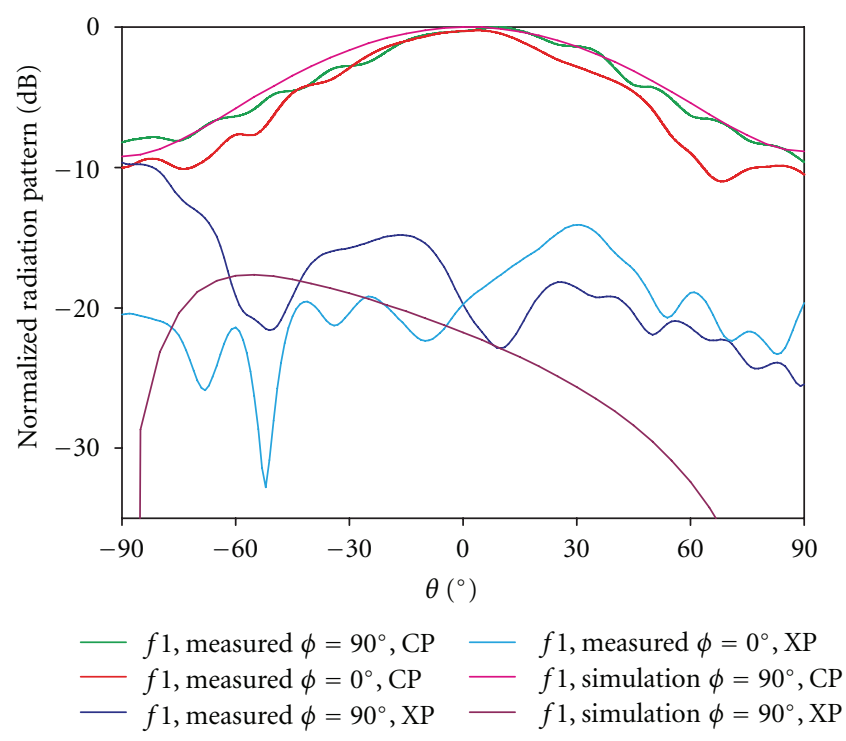

(a)

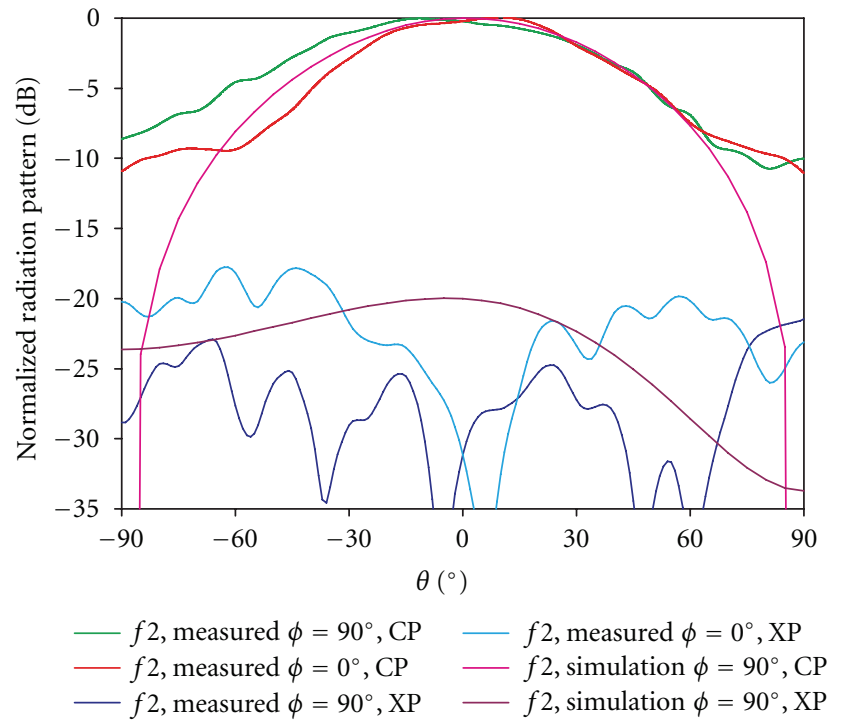

(b)

FIGURE 9: (a) Measured normalized copolar and cross-polar gain radiation patterns for $0^{\circ}$ and $90^{\circ}$ phi cuts. Simulated normalized copular and cross-polar gain radiation pattern for $90^{\circ}$ phi cut at $F 1=4.19 \mathrm{GHz}$. (b) Measured normalized copolar and cross-polar gain radiation patterns for $0^{\circ}$ and $90^{\circ}$ phi cuts. Simulated normalized copular and cross-polar gain radiation pattern for $90^{\circ}$ phi cut at $F 2=4.808 \mathrm{GHz}$.

TABLE 2

\begin{tabular}{lc}
\hline \multicolumn{1}{c}{ Parameters } & Measured results \\
\multicolumn{2}{c}{ Matching characteristics } \\
\hline $\begin{array}{l}\text { Frequency, }(\mathrm{GHz}) \\
\text { Reflection coefficient, }(\mathrm{dB})\end{array}$ & 5.16 \\
$\begin{array}{l}\text { Effective bandwidth at } \\
-5 \mathrm{~dB}(\%)\end{array}$ & -22.3 \\
\hline \multicolumn{1}{c}{ Radiation characteristics } \\
\hline $\begin{array}{l}\text { Peak directivity, (dBi) } \\
\text { Peak gain, (dB) } \\
\text { Radiation efficiency (\%) }\end{array}$ \\
\hline
\end{tabular}

Comparing Tables 1 and 2, the designed prototype presents similar performances to those of the conventional patch antenna from matching point of view (effective bandwidth and reflection coefficient values). On the other side, from radiation point of view, the designed and fabricated dual band antenna resonances show lower radiation efficiencies as it has been mentioned before.

Finally, to understand in an oversimplified way the radiation mechanism of the proposed antenna, one needs to consider the pair of electric dipoles that are described in [9] and the effect of the finite ground plane. In a recent work a refined equivalent circuit model for the CSRR which explains more accurately the physical interpretation of the influence of reactive parameters [19] is presented. This circuit model will be developed in further works in order to take into account the radiation resistance and the internal coupling to the patch and ground plane.

\section{Conclusions}

In this work a dual band patch antenna based on a CSRR has been proposed, studied, and successfully tested, demonstrating the feasibility of this type of dual band antennas, adding a miniaturization of patch dimensions for some locations of the CSRR inside the patch. A good agreement between simulated and measured results is shown. The parametric study shows the influence of the location of the CSRR on the patch on the radiation characteristics. The design of the dual patch antenna is simple as the only design parameters comparing to a conventional path antenna are the ones of the CSRR particle design parameters. Also, multiband antennas can be designed in a similar way by simply adding different CSRRs on the patch.

\section{Acknowledgments}

This work has been supported by the Spanish Government and EU Feder by the Contracts Consolider "Engineering Metamaterials" CSD2008-00066 and TEC2008-06871-C0201.

\section{References}

[1] V. G. Veselago, "The electrodynamics of substances with simultaneously negative values of $\varepsilon$ and $\mu$," Soviet Physics Uspekhi, vol. 10, pp. 509-514, 1968.

[2] D. R. Smith, W. J. Padilla, D. C. Vier, S. C. Nemat-Nasser, and S. Schultz, "Composite medium with simultaneously negative permeability and permittivity," Physical Review Letters, vol. 84, no. 18, pp. 4184-4187, 2000.

[3] J. B. Pendry, A. J. Holden, D. J. Robbins, and W. J. Stewart, "Magnetism from conductors and enhanced nonlinear 
phenomena," IEEE Transactions on Microwave Theory and Techniques, vol. 47, no. 11, pp. 2075-2084, 1999.

[4] R. Marqués, F. Mesa, J. Martel, and F. Medina, "Comparative analysis of edge- and broadside-coupled split ring resonators for metamaterial design-theory and experiments," IEEE Transactions on Antennas and Propagation, vol. 51, no. 10, pp. 2572-2581, 2003.

[5] R. Marqués, F. Medina, and R. Rafii-El-Idrissi, "Role of bianisotropy in negative, permeability and left-handed metamaterials," Physical Review B, vol. 65, no. 14, pp. 14444011444406, 2002.

[6] F. Martín, F. Falcone, J. Bonache, T. Lopetegi, R. Marqués, and M. Sorolla, "Miniaturized coplanar waveguide stopband filters based on multiple tuned split rin resonators," IEEE Microwave and Wireless Components Letters, vol. 13, no. 12, pp. 511-513, 2003.

[7] F. Falcone, F. Martín, J. Bonache, R. Marqués, T. Lopetegi, and M. Sorolla, "Left handed coplanar waveguide band pass filters based on Bi-layer split ring resonators," IEEE Microwave and Wireless Components Letters, vol. 14, no. 1, pp. 10-12, 2004.

[8] F. Falcone, T. Lopetegi, J. D. Baena, R. Marqués, F. Martín, and M. Sorolla, "Effective negative- $\varepsilon$ stopband microstrip lines based on complementary split ring resonators," IEEE Microwave and Wireless Components Letters, vol. 14, no. 6, pp. 280-282, 2004.

[9] F. Falcone, T. Lopetegi, M. A. G. Laso et al., "Babinet principle applied to metasurface and metamaterial design," Physical Review Letters. In press.

[10] R. W. Ziolkowski and A. D. Kipple, "Application of double negative materials to increase the power radiated by electrically small antennas," IEEE Transactions on Antennas and Propagation, vol. 51, no. 10, pp. 2626-2640, 2003.

[11] F. Qureshi, M. A. Antoniades, and G. V. Eleftheriades, "A compact and low-profile metamaterial ring antenna with vertical polarization," IEEE Antennas and Wireless Propagation Letters, vol. 4, no. 1, pp. 333-336, 2005.

[12] R. K. Baee, G. Dadashzadeh, and F. G. Kharakhili, "Using of CSRR and its equivalent circuit model in size reduction of microstrip antenna," in Proceedings of the Asia-Pacific Microwave Conference (APMC '07), pp. 1-4, 2007.

[13] Y. Lee, S. Tse, Y. Hao, and C. G. Parini, "A compact microstrip antenna with improved bandwidth using complementary split-ring resonator (CSRR) loading," in Proceedings of the IEEE Antennas and Propagation Society International Symposium, pp. 5431-5434, June 2007.

[14] L. Meng, L. Mingzhi, and J. C. Tie, "Novel miniaturized dual band antenna design using complementary metamaterial," in Proceedings of the International Workshop on Metamaterials (META '08), pp. 374-376, November 2008.

[15] J. Liu, S. Gong, Y. Xu, X. Zhang, C. Feng, and N. Qi, "Compact printed ultra-wideband monopole antenna with dual bandnotched characteristics," Electronics Letters, vol. 44, no. 12, pp. 710-711, 2008.

[16] H. Zhang, Y. Q. Li, X. Chen, Y. Q. Fu, and N. C. Yuan, "Design of circular/dual-frequency linear polarization antennas based on the anisotropic complementary split ring resonator," IEEE Transactions on Antennas and Propagation, vol. 57, no. 10, Article ID 5196779, pp. 3352-3355, 2009.

[17] K.-L. Wong, Compact and Broadband Microstrip Antennas, John Wiley \& Sons, New York, NY, USA, 2002.

[18] J. D. Kraus and R.J. Marhefka, Antennas for all Applications, McGraw-Hill, New York, NY, USA, 3rd edition, 2002.

[19] F. Aznar, M. Gil, J. Bonache, and F. Martín, "Revising the equivalent circuit models of resonant-type metamaterial transmission lines," in Proceedings of the IEEE MTT-S International Microwave Symposium Digest (MTT '08), pp. 323-326, June 2008. 

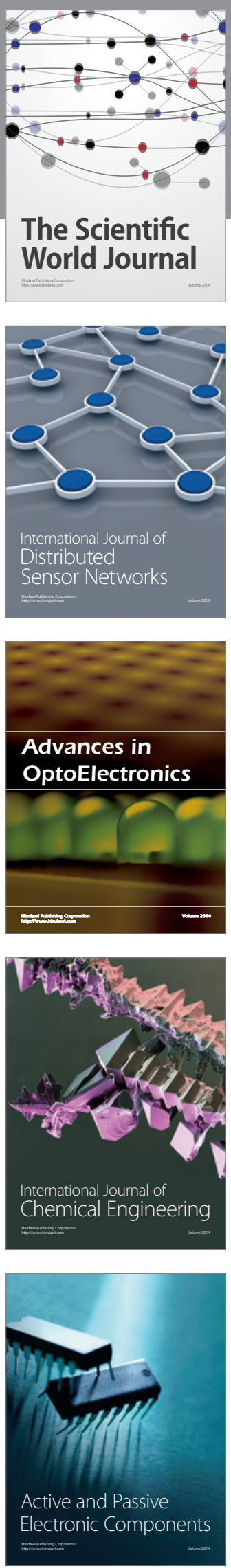
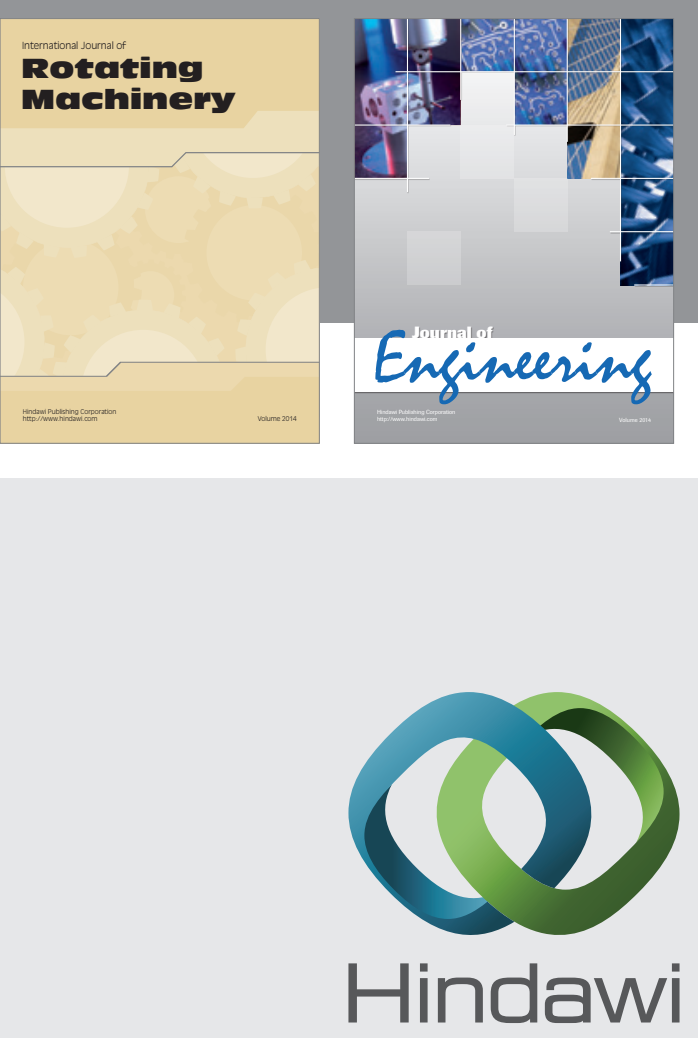

Submit your manuscripts at

http://www.hindawi.com
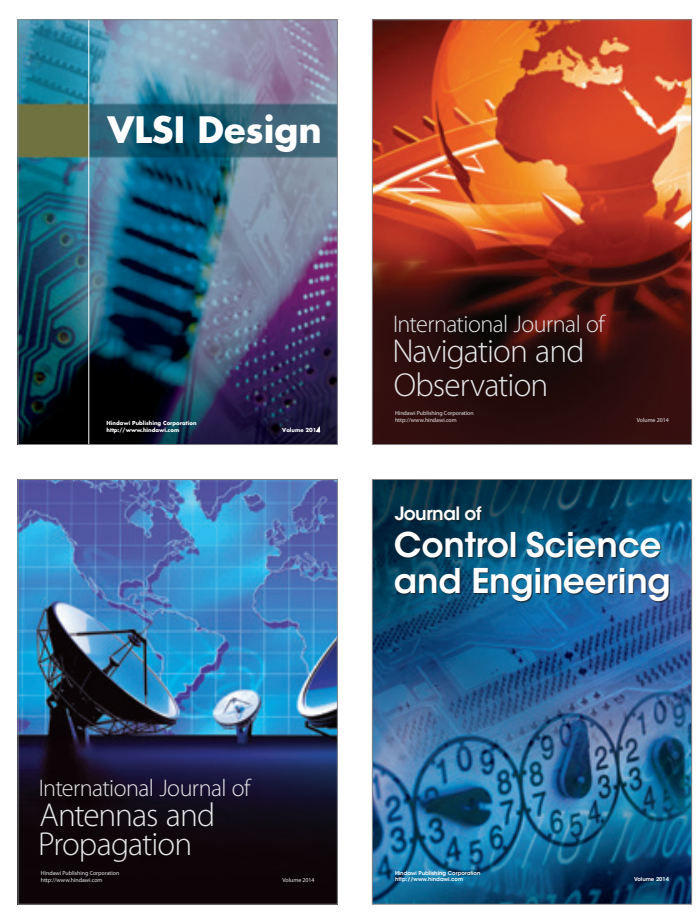
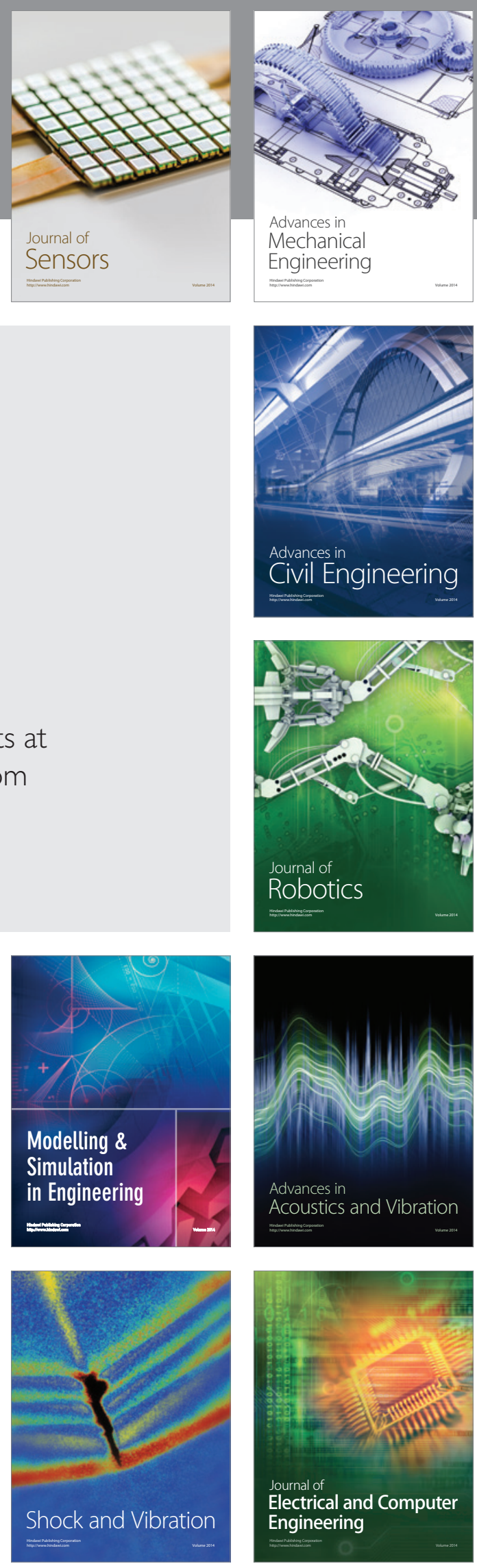